Volume 1

Issue 1 -- Melanoma Research

Article 5

2-28-2014

\title{
The Molecular Biology and Treatment of Malignant Melanoma with BRAFV600 Mutations
}

Michael P. Mullane

Follow this and additional works at: https://aah.org/jpcrr

Part of the Oncology Commons

\section{Recommended Citation}

Mullane MP. The molecular biology and treatment of malignant melanoma with BRAFV600 mutations. J Patient Cent Res Rev. 2014;1:21-26. doi: 10.17294/2330-0698.1007

Published quarterly by Midwest-based health system Advocate Aurora Health and indexed in PubMed Central, the Journal of Patient-Centered Research and Reviews (JPCRR) is an open access, peer-reviewed medical journal focused on disseminating scholarly works devoted to improving patient-centered care practices, health outcomes, and the patient experience. 


\title{
The Molecular Biology and Treatment of Malignant Melanoma with BRAF $^{\text {v600 }}$ Mutations
}

\author{
Michael P. Mullane, MD \\ Department of Oncology/Hematology, Aurora Advanced Healthcare, Racine, WI
}

\section{Abstract}

Since 2011, the treatment options for metastatic malignant melanoma have significantly changed. In that year, ipilimumab, an anti-CTLA4 monoclonal antibody, and vemurafenib, a potent inhibitor of mutated-BRAF (V600E and V600K), were approved by the FDA. In 2013, dabrafenib, another inhibitor of mutated-BRAF, and trametinib, a MEK inhibitor, were approved by the FDA. Most recently, combination therapy with dabrafenib and trametinib was approved. This article will describe a patient with metastatic malignant melanoma with $\mathrm{BRAF}^{\mathrm{V} 600 \mathrm{E}}$ who has responded very well to vemurafenib monotherapy. We will then explore the molecular basis, pharmacologic development and clinical outcomes of inhibition of the mitogen-activated protein (MAP) kinase pathway in patients with metastatic malignant melanoma with oncogenic BRAF (V600E and V600K). (J Patient-Centered Res Rev. 2014;1(1):21-26.)

\section{Keywords}

melanoma, targeted therapy, mutation, mitogen-activated protein kinase

\section{Clinical Case}

CA is a 69-year-old woman who presented in September 2010 with a changing pigmented lesion on her right lower extremity. A punch biopsy was positive for malignant melanoma. The patient underwent a wide local excision and sentinel lymph node biopsy on October 28, 2010. Pathology demonstrated a stage IIA nodular malignant melanoma (pT3a pN0sn M0). All margins were widely negative. The patient was observed and did well until April 2011. At that time she developed multiple in-transit metastases involving her right lower extremity. Extensive staging studies showed no evidence of regional adenopathy or extra regional metastatic disease. Molecular studies showed mutated BRAF (V600E). She underwent serial resection of in-transit metastases on a weekly basis from April 7, 2011, to October 5, 2011. Each

Correspondence: Michael P. Mullane, MD

Aurora Cancer Care, 1151 Warwick Way,

Racine, WI 53405 Phone: 262-886-7360,

Fax: 262-866-7361, Email: michael.mullane@aurora.org week multiple new in-transit metastases were identified and resected. By mid-October there were more than 15 new intransit metastases. In addition, restaging studies showed multiple new pulmonary nodules consistent with metastatic disease. The patient started vemurafenib $960 \mathrm{mg}$ orally twice daily on October 26, 2011. Since starting vemurafenib, no new in-transit metastases have been identified. She has had a significant partial response to vemurafenib, with reduction in the size and number of pulmonary metastases and regression of all in-transit metastases. She has tolerated vemurafenib well.

\section{Introduction}

The prognosis of patients diagnosed with metastatic malignant melanoma is dismal. Up until recently, treatment options consisted of high-dose interleukin-2 in a small select group of patients, cytotoxic chemotherapy or biochemotherapy with very limited clinical benefit or best supportive care. Recently, immunotherapy with ipilimumab, an anti-CTLA4 monoclonal antibody, and inhibition of the mitogen-activated protein (MAP) kinase pathway in patients whose tumors contain a $\mathrm{BRAF}^{\mathrm{V} 600}$ mutation have shown significant clinical benefit. This article will focus on the clinical features and molecularly targeted therapy of metastatic malignant melanoma containing $\mathrm{BRAF}^{\mathrm{V} 600}$ mutations.

The MAP kinase signal transduction pathway regulates a number of physiologic responses including cell proliferation. In the absence of mutations the pathway is activated by ligand binding to the extracellular domain of a receptor tyrosine kinase (RTK). Ligand-bound RTK leads to RTK dimerization and autophosphorylation. This leads to activation of RAS protein through the exchange of GDP for GTP. Activated RAS activates RAF proteins (ARAF, BRAF and CRAF). Activated RAF phosphorylates and activates MEK, which in turn phosphorylates and activates ERK. Activated ERK activates transcription factors that lead to the expression of many genes, resulting in a number of effects including cell proliferation. The MAP kinase pathway is deregulated in as many as one-third of human cancers, and as such, components of this pathway (particularly RAS, RAF 
and MEK) have been identified as important therapeutic targets. ${ }^{1}$ The RAS-RAF-MEK-ERK pathway is activated in the majority of malignant melanoma cases. ${ }^{2}$ There has been significant scientific effort to evaluate the various components of this pathway and identify mechanisms of MAP kinase pathway activation in human cancers in general and in malignant melanoma specifically. In addition, recent whole-exome sequencing studies have identified a number of other driver mutations in malignant melanoma. Further characterization of these mutations and their pathways may lead to other therapeutic targets. ${ }^{3,4}$

\section{Discovery of BRAFv600 Mutations}

Because of the importance of the MAP kinase pathway in human cancer, Davies et al. screened more than 500 cancer cell lines for the presence of mutations in RAF genes. All probable oncogenic mutations were found in $B R A F$ in the kinase domain in exons 11 and 15. These two exons were then screened for mutations in genomic DNA from a wide range of human cancers and short-term cultures derived from human cancers. They demonstrated BRAF somatic missense mutations in $66 \%$ of malignant melanomas and a lower frequency of mutations in a number of other human cancers. The mutation $\mathrm{V} 600 \mathrm{E}$ accounted for $80 \%$ of the mutations identified. The significance of these mutations were underscored by the demonstration that mutated BRAF proteins have elevated kinase activity, are transforming in NIH 3T3 cells, and growth of cancer cell lines with the V600E mutation is independent of RAS function. These observations led the authors to postulate that inhibition of BRAF activity may be an important therapeutic strategy in the treatment of metastatic melanoma. ${ }^{5}$

\section{Prevalence of BRAFv600 Mutations in Different Melanoma Subtypes}

A number of early small studies showed a high frequency of BRAF mutations in malignant melanoma. BRAF mutations were found in highest frequency in cutaneous melanoma. Acral and mucosal melanomas have much lower frequency BRAF mutations but have distinct alternate patterns of genetic alterations. ${ }^{1,6,7}$ The largest study to date reported clinical $B R A F$ testing results in a retrospective analysis of 1,112 specimens of primary and metastatic melanoma at the University of Texas MD Anderson Cancer Center obtained from January 2008 through August 2011. Mutated BRAF was identified in 499 specimens (45\%). Nonacral cutaneous melanoma comprised the largest subset $(n=774)$, of which $51 \%$ contained a BRAF mutation with no difference between primary and metastatic sites. BRAF mutations were found in all cutaneous histological subtypes. Superficial spreading melanoma had the highest frequency of BRAF mutations $(66 \%)$. BRAF mutations also were found in nodular melanoma (55\%) and lentigo maligna melanoma (20\%). Acral cutaneous melanoma $(n=111)$ had BRAF mutations in $16 \%$ of specimens. BRAF mutations were identified in $47 \%$ of metastatic melanoma of unknown primary $(n=177)$. There were no BRAF mutations identified in mucosal $(n=26)$, uveal $(n=23)$ or primary leptomeningeal $(n=1)$ sites. The $\mathrm{BRAF}^{\mathrm{V} 600 \mathrm{E}}$ mutation accounted for $75 \%$ of BRAF mutations $(n=376)$. The $\mathrm{BRAF}^{\mathrm{V} 600 \mathrm{~K}}$ mutation $(17 \%, \mathrm{n}=86)$ and other V600 mutations $(5 \%, n=23)$ accounted for the majority of the rest of the BRAF mutations. ${ }^{8}$

\section{Nonselective BRAF Inhibition With Sorafenib}

Sorafenib is a multikinase inhibitor that, among other actions, inhibits the MAP kinase pathway at the level of RAF kinase. ${ }^{9}$ In early-phase single-agent clinical studies of sorafenib, which included patients with advanced malignant melanoma, sorafenib was well tolerated but associated with low response rates. ${ }^{10,11}$ In a phase I trial of carboplatin and paclitaxel with sorafenib conducted in patients with advanced solid tumors, the majority of which were advanced refractory melanoma, modest activity was observed. ${ }^{12}$ These results led to two large randomized phase III trials to test the activity of sorafenib in combination with carboplatin and paclitaxel in first- or second-line therapy. Both studies showed no benefit from the addition of sorafenib to combination chemotherapy. ${ }^{13,14}$ These studies were conducted with unselected patients with no molecular inclusion criteria. In addition, there was no attempt to demonstrate MAP kinase pathway inhibition. It is possible there was some clinical activity in specific molecularly defined subgroups. However, at present, no clinically meaningful activity for sorafenib as a single agent or in combination with chemotherapy in the treatment of metastatic melanoma has been demonstrated.

\section{Development of the Specific BRAF Inhibitor} Vemurafenib and Clinical Experience With Vemurafenib and Dabrafenib

In 2008, Tsai et al. reported the design of a highly potent and specific inhibitor of the $\mathrm{BRAF}^{\mathrm{V} 600 \mathrm{E}}$ gene product. This compound (PLX4720) was developed using a scaffold-and structure-based rational drug design of a kinase inhibitor specific to the BRAF ${ }^{\mathrm{V} 600 \mathrm{E}}$ kinase. They demonstrated that ERK phosphorylation was inhibited in tumor cell lines containing the BRAF ${ }^{\mathrm{V} 600 \mathrm{E}}$ but not in cells lacking oncogenic BRAF mutations. In tumor xenograft models performed with a BRAFV600E containing cell line in nude mice orally dosed PLX4720 had significant antitumor activity without evidence of toxicity. ${ }^{15}$ PLX4032 (vemurafenib), an analogue 
of PLX4720, also was shown to be a highly potent and specific inhibitor of oncogenic BRAF kinase. ${ }^{16}$ This compound was taken into clinical trials.

A multicenter phase I dose escalation trial of PLX4032 followed by an extension trial using the recommended phase II dose demonstrated significant clinical activity in patients with metastatic malignant melanoma with tumors that harbor the $\mathrm{BRAF}^{\mathrm{V} 600 \mathrm{E}}$ mutation. There were 32 patients in the extension cohort, all of whom received the recommended phase II dose of $960 \mathrm{mg}$ twice daily. Among these patients, 24 had a partial response and 2 had a complete response. At the time of the report the estimated median progressionfree survival among all patients was more than 7 months. ${ }^{17}$ A subsequent phase III randomized clinical trial compared vemurafenib (PLX4032) with dacarbazine. All patients had previously untreated metastatic melanoma with BRAFV600E mutation. A total of 2,107 patients underwent screening, yielding 675 eligible patients who were randomly assigned in a 1:1 ratio to receive either vemurafenib $(960 \mathrm{mg}$ orally twice daily) or dacarbazine $\left(1,000 \mathrm{mg} / \mathrm{m}^{2}\right.$ intravenously every 3 weeks). The primary end points were rates of overall and progression-free survival. Secondary end points were response rate, response duration and safety. Vemurafenib was associated with a statistically significant relative reduction in the risk of death (hazard ratio [HR] 0.37, 95\% confidence interval [CI] 0.26-0.55; $\mathrm{P}<0.001)$ and the risk of tumor progression (HR 0.26, 95\% CI 0.20-0.33; $\mathrm{P}<0.001$ ). There was a significant difference in confirmed objective responses in patients treated with vemurafenib compared with those treated with dacarbazine ( $48 \%$ vs. $5 \%$; $<0.001)$. The estimated median progression-free survival was 5.3 months in the vemurafenib group and 1.6 months in the dacarbazine group. ${ }^{18}$ On August 17, 2011, the U.S. Food and Drug Administration (FDA) approved vemurafenib for the treatment of advanced malignant melanoma harboring the BRAF ${ }^{\mathrm{V} 600 \mathrm{E}}$ mutation. Similar results were found in a randomized controlled trial of dabrafenib, another inhibitor of mutated BRAF, versus dacarbazine, leading to FDA drug approval on May 29, 2013. ${ }^{19}$

Inhibition of oncogenic BRAF also has been shown to have activity in patients with central nervous system (CNS) metastases. Long et al. reported the results of an openlabel phase II trial of dabrafenib in patients with metastatic melanoma with at least one asymptomatic brain metastasis. All patients had histologically confirmed melanoma containing mutated BRAF (V600E or V600K). There were two groups of patients treated. Cohort A had not received previous local CNS-directed therapy $(\mathrm{n}=89)$, and Cohort $\mathrm{B}$ had progressive brain metastases after previous local therapy $(\mathrm{n}=83)$. Patients were treated with oral dabrafenib $(150 \mathrm{mg}$ twice daily) until disease progression, death or unacceptable adverse events. Of the 139 patients with BRAF $^{6600 E}$, objective response was identified in 29 of 74 patients (39\%) in Cohort A and 20 of 65 patients (31\%) in Cohort B. The overall disease control rate (complete response + partial response + stable disease) was $80 \%$ in Cohort A (95\% CI: 68.8-88.2) and $83 \%$ in Cohort B (95\% CI: 71.7-91.2). The estimated 6-month survival was 61\% in Cohort A (95\% CI: 46.7-73.2) and 61\% in Cohort B (95\% CI: 46.3-72.7). There were only 5 objective responses noted in the 33 patients (15\%) with BRAF ${ }^{\mathrm{V} 600 \mathrm{~K}}$ (1 of 15 in Cohort A and 4 of 18 in Cohort B). In addition to an adverse event profile similar to other studies involving mutant-BRAF inhibitors, there were $10(6 \%)$ episodes of intracranial hemorrhage, one of which was treatment related..$^{20}$ There are also case reports of activity with vemurafenib in the treatment of malignant melanoma with brain metastases. ${ }^{21}$ There are no studies to date that directly compare mutant-BRAF inhibition to radiation therapy. Nor are there any studies that evaluate concurrent or sequential combined radiation therapy and mutant-BRAF inhibition.

Although inhibition of mutant BRAF with vemurafenib or dabrafenib results in significantly greater response rates and improved progression-free and overall survival in patients with metastatic malignant melanoma whose tumors have a V600 mutation, virtually all patients will progress. Patients progress after a median duration of just 7 months of therapy. Interestingly, identified mechanisms of resistance do not include loss of the $\mathrm{BRAF}^{\mathrm{V} 600}$ mutation or additional mutations within BRAF. In many patients at progression, MAP kinase signaling is again upregulated, bypassing mutant-BRAF inhibition. ${ }^{22}$ Several mechanisms of resistance resulting in restoration of MEK activation have been described. Examples include acquired mutations in $N R A S$, which activates MEK via CRAF, RAF independent activation of MEK via MAP3K8/COT, acquired activating MEK mutations, $\mathrm{BRAF}^{\mathrm{V} 600 \mathrm{E}}$ gene amplification, and BRAF alternative gene splicing. ${ }^{23,24,25,26}$ Based on the known series of activation events in the MAP kinase pathway and on some of the mechanisms of acquired resistance identified in mutant-BRAF inhibition, targeting downstream events (MEK or ERK) or combination inhibition was hypothesized to have a clinical benefit. There are MAP kinase-independent mechanisms of resistance that have been identified as well. New therapeutic strategies will be required to address these resistance mechanisms. 
Single-Agent MEK Inhibition and Combination MEK/Mutant-BRAF Inhibition

Trametinib is a potent selective inhibitor of MEK1/MEK2. Significant clinical activity for trametinib was demonstrated in patients with metastatic malignant melanoma with a $\mathrm{BRAF}^{\mathrm{V} 600 \mathrm{E}}$ or $\mathrm{BRAF}^{\mathrm{V} 600 \mathrm{~K}}$ mutation not previously treated with a BRAF inhibitor when compared to chemotherapy (either dacarbazine or paclitaxel). In a phase III openlabel trial, 322 such patients were randomly assigned to receive either trametinib or chemotherapy in a $2: 1$ ratio. Progression-free and overall survival were the primary and secondary end points. Patients progressing on chemotherapy were permitted to crossover to receive trametinib. Patients receiving trametinib had improved progression-free survival compared with chemotherapy (4.8 months vs. 1.5 months; HR: $0.45 ; 95 \%$ CI: $0.33-0.63 ; \mathrm{P}<0.001)$. Despite the crossover design, patients receiving trametinib had improved overall survival at 6 months compared with chemotherapy (81\% vs. $67 \%$; HR: $0.54 ; 95 \%$ CI: 0.32-0.92; $\mathrm{P}=0.01) .{ }^{27}$ On May 29, 2013, the FDA approved trametinib for use in patients with metastatic melanoma with $\mathrm{BRAF}^{\mathrm{V} 600 \mathrm{E}}$ or $\mathrm{BRAF}^{\mathrm{V} 600 \mathrm{~K}}$ mutations. The approval did not extend to patients previously treated with a BRAF inhibitor.

To explore the hypothesis that the addition of MEK inhibition to inhibition of mutated BRAF would result in improved clinical activity in the treatment of patients with metastatic melanoma with $\mathrm{BRAF}^{\mathrm{V} 600}$ mutations, Flaherty and colleagues performed a four-part phase I/II openlabel trial of trametinib in combination with dabrafenib. In part $\mathrm{C}$ of the trial, 162 patients were randomly assigned in a 1:1:1 ratio to receive dabrafenib alone $(150 \mathrm{mg}$ orally twice daily) or in combination with trametinib (1 or $2 \mathrm{mg}$ orally once daily). Patients who progressed on dabrafenib monotherapy could crossover to the higher dose combination therapy (150/2). Patients receiving the higher dose combination therapy (150/2) had significantly better median progression-free survival compared with dabrafenib monotherapy (9.4 months vs. 5.8 months; HR: 0.39; 95\% CI: $0.25-0.62 ; \mathrm{P}<0.001)$. This difference was less pronounced, although still statistically significant, after overview by an independent review committee. Progression-free survival at 1 year was significantly greater in the combination $150 / 2$ group as compared to monotherapy ( $41 \%$ vs. $9 \%$; $\mathrm{P}<0.001)$. The overall response rate and duration of response were greater in the combination $150 / 2$ group as compared to monotherapy ( $76 \%$ vs. $54 \%[\mathrm{P}=0.03]$ and 10.5 months $[95 \%$ CI: 7.4-14.9] vs. 5.6 months [95\% CI: 4.5-7.4]). The lower dose combination therapy (150/1) was less effective than the higher dose combination (150/2) but still significantly superior than dabrafenib monotherapy. ${ }^{28}$ Data presented in abstract form at the 2013 European Cancer Congress showed similar activity for the combination of vemurafenib and cobimetinib (a MEK inhibitor). ${ }^{29}$ On January 9, 2014, the FDA approved trametinib in combination with dabrafenib for use in patients with metastatic melanoma with $\mathrm{BRAF}^{\mathrm{V} 600 \mathrm{E}}$ or $\mathrm{BRAF}^{\mathrm{V} 600 \mathrm{~K}}$ mutations.

Interestingly, single-agent MEK inhibition with trametinib has no activity in patients with BRAF-mutated metastatic melanoma progressing after treatment with a BRAF inhibitor. It is likely that resistance mechanisms to inhibition of mutated BRAF also confer resistance to MEK inhibition. ${ }^{30}$ In addition, combination mutant-BRAF and MEK inhibition after progression on mutant-BRAF inhibitors has only modest activity. ${ }^{29}$ Taken together, these data suggest that patients with metastatic BRAF-mutant melanoma previously untreated with BRAF inhibitors have an improved clinical outcome when treated with concurrent combination mutantBRAF and MEK inhibition as compared to mutant-BRAF inhibition alone or sequential use of mutant-BRAF and MEK inhibitors. It appears likely that this improved activity is superior to single-agent MEK inhibition as well. Phase III trial data will be available soon to assess for improvement in overall survival.

\section{Cutaneous Squamous Cell Carcinoma Associated} With Mutant-BRAF Inhibition

Toxicities associated with single-agent or combination mutant-BRAF/MEK inhibition are not reviewed here. However, a well-described cutaneous adverse event of mutant-BRAF inhibition is the development of cutaneous squamous cell carcinoma and keratoacanthoma in $15-30 \%$ of treated patients. Mutations in RAS are frequently identified in these lesions. The mechanism of action is likely related to paradoxical activation of the MAP kinase pathway through CRAF in BRAF wild-type cells that have mutated RAS or upregulated RTKs. ${ }^{31}$ In patients treated with combination mutant-BRAF/MEK inhibition, the risk of squamous cell carcinoma and keratoacanthoma is significantly reduced, consistent with inhibition of paradoxical MAP kinase activation in BRAF wild-type cells through the inhibition of MEK. In the combination study reported by Flaherty, patients receiving dabrafenib monotherapy had an incidence of cutaneous squamous cell carcinoma and keratoacanthoma of $19 \%$. The incidence of these lesions was $7 \%$ in patients treated with the higher dose combination dabrafenib and trametinib therapy $(150 / 2)$ and $2 \%$ in patients with the lower dose combination therapy $(150 / 1)$. These results were statistically significant $(\mathrm{P}=0.004$ and $\mathrm{P}=0.09$, respectively $) .{ }^{28}$ 


\section{Conclusions}

The rapidly evolving understanding of malignant cell proliferation has created an ever-expanding array of therapeutic targets to exploit. The importance of oncogenic BRAF in malignant melanoma was identified in the landmark paper published in 2002 by Davies et al. In spite of early negative studies with nonspecific BRAF inhibitors, intense scientific study of the MAP kinase pathway in malignant melanoma persisted. By 2008, specific potent inhibitors of mutant BRAF were developed and taken to clinical trials. The FDA approved mutant-BRAF inhibitors for the treatment of unresectable or metastatic malignant melanoma associated with BRAF $^{\mathrm{V} 600}$ mutations in 2011 (vemurafenib) and 2013 (dabrafenib). In 2013, the FDA also approved a MEK inhibitor for the treatment of these patients. Monotherapy with mutant-BRAF or MEK inhibitors represents a significant advance in the treatment of metastatic melanoma. However, the majority of these patients develop resistance to monotherapy and progress within 1 year of therapy initiation. While we wait for mature phase III data, early reports from combination mutant-BRAF and MEK inhibition show very promising results, which led the FDA to approve combination therapy (dabrafenib and trametinib) in January 2014. Unfortunately, even with these new agents, the majority of patients with metastatic malignant melanoma will eventually die of their disease. The roles of these agents in earlier stages of melanoma are now being explored. Additional evaluation of other targets in the MAP kinase pathway (e.g. ERK) and further understanding and targeting of alternative bypass pathways as well as exploration of further therapeutic combinations (multiple inhibitors with or without anti-CTLA4 therapy) will hopefully further improve clinical results.

Conflicts of Interest

None. 


\section{References}

1 Dhillon AS, Hagan S, Rath O, et al. MAP kinase signaling pathways in cancer. Oncogene. 2007;26:3279-3290.

2 Omholt K, Platz A, Kanter L, et al. NRAS and BRAF mutations arise early during melanoma pathogenesis and are preserved throughout tumor progression. Clin Cancer Res. 2003;9:6483-6488.

3 Krauthammer M, Kong Y, Ha BH, et al. Exome sequencing identifies recurrent somatic RAC1 mutations in melanoma. Nat Genet. 2012;44:1006-1014.

4 Hodis E, Watson IR, Kryukov GV, et al. A landscape of driver mutations in melanoma. Cell. 2012;150:251-263.

5 Davies H, Bignell GR, Cox C, et al. Mutations of the BRAF gene in human cancer. Nature. 2002;417:949-954.

6 Rubenstein JC, Sznol M, Pavlick AC, et al. Incidence of the V600K mutation among melanoma patients with BRAF mutations and potential therapeutic response to the specific BRAF inhibitor PLX4032. J Transl Med. 2010;8:67.

7 Curtin JA, Fridlyand J, Kageshita T, et al. Distinct sets of genetic alterations in melanoma. $N$ Engl J Med. 2005;353:2135-2147.

8 Greaves WO, Verma S, Patel KP, et al. Frequency and spectrum of BRAF mutations in a retrospective, single-institution study of 1112 cases of melanoma. J Mol Diagn. 2013;15:220-226.

9 Wilhelm SM, Carter C, Tang L, et al. BAY 43-9006 exhibits broad spectrum oral antitumor activity and targets the RAF/MEK/ERK pathway and receptor tyrosine kinases involved in tumor progression and angiogenesis. Cancer Res. 2004;64:7099-7109.

10 Strumberg D, Richly H, Hilger RA, et al. Phase I clinical and pharmacokinetic study of the novel RAF kinase and vascular endothelial growth factor receptor inhibitor BAY 43-9006 in patients with advanced refractory solid tumors. J Clin Oncol. 2005;23:965972.

11 Eisen T, Ahmad T, Flaherty KT, et al. Sorafenib in advanced melanoma: a Phase II randomised discontinuation trial analysis. Br J Cancer. 2006;95:581-586.

12 Flaherty KT, Brose M, Schuchter LM, et al. Sorafenib combined with carboplatin and paclitaxel for metastatic melanoma: Progressionfree survival and response versus B-RAF status. (abstr.) Ann Oncol. 2006;17(suppl 3):iii33.

13 Hauschild A, Agarwala SS, Trefzer U, et al. Results of a phase III, randomized, placebo-controlled study of sorafenib in combination with carboplatin and paclitaxel as second-line treatment in patients with unresectable stage III or stage IV melanoma. J Clin Oncol. 2009;27:2823-2830.

14 Flaherty KT, Lee SJ, Schuchter LM, et al. Final results of E2603: A double-blind, randomized phase III trial comparing carboplatin $(\mathrm{C}) /$ paclitaxel $(\mathrm{P})$ with or without sorafenib $(\mathrm{S})$ in metastatic melanoma. (abstr.) J Clin Oncol. 2010;28(15 suppl):8511.

15 Tsai J, Lee JT, Wang W, et al. Discovery of a selective inhibitor of oncogenic B-Raf kinase with potent antimelanoma activity. Proc Natl Acad Sci U S A. 2008;105:3041-3046.

16 Søndergaard JN, Nazarian R, Wang Q, et al. Differential sensitivity of melanoma cell lines with BRAFV600E mutation to the specific Raf inhibitor PLX4032. J Transl Med. 2010;8:39.

17 Flaherty KT, Puzanov I, Kim KB, et al. Inhibition of mutated, activated BRAF in metastatic melanoma. $N$ Engl J Med. 2010;363:809-819.

18 Chapman PB, Hauschild A, Robert C, et al. Improved survival with vemurafenib in melanoma with BRAF V600E mutation. $N$ Engl $J$ Med. 2011;364:2507-2516.

19 Hauschild A, Grob JJ, Demidov LV, et al. Dabrafenib in BRAFmutated metastatic melanoma: a multicentre, open-label, phase 3 randomised controlled trial. Lancet. 2012;380:358-365.
20 Long GV, Trefzer U, Davies MA, et al. Dabrafenib in patients with Val600Glu or Val600Lys BRAF-mutant melanoma metastatic to the brain (BREAK-MB): a multicentre, open-label, phase 2 trial. Lancet Oncol. 2012;13:1087-1095.

21 Dummer R, Rinderknecht J, Goldinger SM, et al. An open-label pilot study of vemurafenib in previously treated metastatic melanoma patients with brain metastases. (abstr.) J Clin Oncol. 2011;29(15 suppl):8548.

22 Sosman JA, Pavlick AC, Schuchter LM, et al. Analysis of molecular mechanisms of response and resistance to vemurafenib (vem) in BRAFV600E melanoma. (abstr.) $J$ Clin Oncol. 2012;30(15 suppl):8503.

23 Johannessen CM, Boehm JS, Kim SY, et al. COT drives resistance to RAF inhibition through MAP kinase pathway reactivation. Nature. 2010;468:968-972.

24 Nazarian R, Shi H, Wang Q, et al. Melanomas acquire resistance to B-RAF(V600E) inhibition by RTK or N-RAS upregulation. Nature. 2010;468:973-977.

25 Shi H, Moriceau G, Kong X, et al. Melanoma whole-exome sequencing identifies (V600E)B-RAF amplification-mediated acquired B-RAF inhibitor resistance. Nat Commun. 2012;3:724.

26 Poulikakos PI, Persaud Y, Janakiraman M, et al. RAF inhibitor resistance is mediated by dimerization of aberrantly age and apparently spliced BRAF(V600E). Nature. 2011;480:387-390.

27 Flaherty KT, Robert C, Hersey P, et al. Improve survival with MEK inhibition in BRAF-mutated melanoma. NEngl JMed. 2012;367:107114.

28 Flaherty KT, Infante JR, Daud A, et al. Combined BRAF and MEK inhibition in melanoma with BRAF V600 mutations. $N$ Engl J Med. 2012;367:1694-1703.

29 McArthur G, Gonzalez R, Pavlick A, et al. Vemurafenib (VEM) and MEK inhibitor, cobimetinib (GDC-0973), in advanced BRAFV600mutated melanoma (BRIM7): dose-escalation and expansion results of a phase IB study. (abstr.) Eur J Cancer. 2013;49(suppl 2):S856.

30 Kim KB, Kefford R, Pavlick AC, et al. Phase II study of the MEK1/ MEK2 inhibitor Trametinib in patients with metastatic BRAF-mutant cutaneous melanoma previously treated with or without a BRAF inhibitor. J Clin Oncol. 2013;31:482-489.

$31 \mathrm{Su}$ F, Viros A, Milagre C, et al. RAS mutations in cutaneous squamous cell carcinomas in patients treated with BRAF inhibitors. $N$ Engl $J$ Med. 2012;366:207-215.

(C) 2014 Aurora Health Care, Inc. 